\title{
Correlation Between Enhancement Patterns on Transabdominal Ultrasound and Survival for Pancreatic Ductal Adenocarcinoma
}

\author{
Tongtong Zhou, ${ }^{1,2, *}$ Li Tan, ${ }^{1}, *$ \\ Yang Gui,' Jing Zhang,' \\ Xueqi Chen,' Menghua Dai, ${ }^{3}$ \\ Mengsu Xiao,' Qing Zhang,' \\ Xiaoyan Chang, ${ }^{4}$ Qun Xu, ${ }^{5}$ \\ Chunmei Bai, ${ }^{6}$ Yuejuan Cheng, ${ }^{6}$ \\ Qiang $\mathrm{Xu},{ }^{3}$ Xue Wang, ${ }^{7}$ \\ Hua Meng, ' Wanying Jia,' \\ Ke Lv, (D) Yuxin Jiang
}

'Department of Ultrasound, Peking Union Medical College Hospital, Peking Union Medical College, Chinese Academy of Medical Sciences, Beijing, People's Republic of China;

${ }^{2}$ Department of Ultrasound, China-Japan Friendship Hospital, Beijing, People's Republic of China; ${ }^{3}$ Department of General Surgery,

Peking Union Medical College Hospital, Peking Union Medical College, Chinese Academy of Medical Sciences, Beijing, People's Republic of China; ${ }^{4}$ Department of Pathology, Peking Union Medical College Hospital, Peking Union Medical College, Chinese Academy of Medical Sciences, Beijing, People's Republic of China;

${ }^{5}$ Department of Epidemiology and Biostatistics, Institute of Basic Medical Sciences Chinese Academy of Medical Sciences, School of Basic Medicine Peking Union Medical College, Beijing People's Republic of China; ${ }^{6}$ Department of Medical Oncology, Peking Union Medical College Hospital, Peking Union Medical College, Chinese Academy of Medical Sciences, Beijing, People's Republic of China; ${ }^{7}$ Key Laboratory of Endocrinology of Nationa Health Commission, Department of Endocrinology, Peking Union Medical College Hospital, Chinese Academy of Medical Science and Peking Union Medical College, Beijing, People's Republic of China

*These authors contributed equally to this work

Correspondence: Ke Lv; Yuxin Jiang Department of Ultrasound, Peking Union Medical College Hospital, Peking Union Medical College, Chinese Academy of Medical Sciences, Beijing, People's Republic of China

Tel/Fax +86 I0-69|55493; +86 I0-69|5549|

Email Ivke@I63.com;

yuxinjiangxh@I63.com
Objective: Investigate the CEUS enhancement patterns of PDAC and analyse correlations between the CEUS enhancement pattern and both the degree of tumour tissue differentiation and overall survival (OS).

Methods: The study included 56 patients with locally advanced PDAC, performed conventional ultrasound and CEUS, and analysed characteristics of the CEUS enhancement patterns. In addition, clinical data, such as serum level of CA19-9, TNM stage were collected, and patients' survival times were followed up. TICs of dynamic CEUS images were acquired using image processing software to obtain the peak, TP, sharpness, and AUC. Correlations of the CEUS enhancement patterns of PDAC with the degree of differentiation of tumour tissue and OS were quantitatively analysed, as were the correlations of the TIC parameters and CEUS enhancement patterns with OS.

Results: Enhancement in the arterial phase included iso-enhancement (30.3\%) and hypoenhancement $(69.6 \%)$, and was not significantly correlated with sex, age at disease onset, or lesion size. Also was not significantly correlated with tumour tissue differentiation. Clear survival times were obtained for 50 patients during follow-up, and the median survival time was significantly longer for the patients with iso-enhancement than hypo-enhancement. Among the TIC parameters, peak $_{\text {tumour, }}$ sharpness $_{\text {tumour, AEsharpness, and REsharpness }}$ differed significantly between the group with iso-enhancement and hypo-enhancement $(\mathrm{p}<$ $0.05)$.

Conclusion: The CEUS enhancement patterns of PDAC in the arterial phase include isoenhancement and hypo-enhancement. Enhancement pattern was not significantly correlated with the degree of differentiation of tumour tissue, but patient survival time differed significantly between the two enhancement patterns, with longer survival for patients with iso-enhancement.

Keywords: contrast-enhanced ultrasound, pancreatic ductal adenocarcinoma, survival time, enhancement pattern, time-intensity curve

\section{Introduction}

Pancreatic ductal adenocarcinoma (PDAC) is a malignant tumour with a high mortality rate, and cancer treatment and survivorship statistics (2016) show that the 5-year survival rate for pancreatic malignant tumours is only $7 \% .{ }^{1}$ Due to the early appearance of peripheral tissue infiltration and envelopment of vessels, surgical treatment is only feasible for $20 \%$ of patients. ${ }^{2}$ Imaging has become an indispensable tool for the clinical diagnosis and efficacy evaluation of PDAC. Among imaging techniques, contrast-enhanced ultrasound (CEUS) not only has 
high sensitivity (90\%) and specificity $(88 \%)$ in the diagnosis of PDAC $^{3}$ but also can be used to assess the distribution of blood vessels in the tumour, facilitate pancreatic biopsy, and evaluate the efficacy of chemotherapy. ${ }^{4}$ Quantitative analysis of CEUS can increase the objectivity of diagnosis.

In this study, the characteristics of the enhancement patterns of PDAC determined using conventional ultrasound and CEUS were observed to quantitatively analyse correlations of the enhancement patterns of PDAC with the degree of differentiation of tumour tissue and overall survival (OS).

\section{Materials and Methods}

\section{Patient Selection}

Due to the retrospective nature of the study, ethics approval was waived by our ethics board. From January 2014 to December 2017, 56 patients received ultrasound-guided biopsy of pancreatic space-occupying lesions in the Department of Medical Ultrasound at Peking Union Medical College Hospital. The inclusion criteria were as follows: 1) the patient's agreement to undergo CEUS examination, signing of informed consent, and completion of CEUS before treatment; 2) pathological confirmation of PDAC by ultrasoundguided percutaneous pancreatic biopsy; and 3) a diagnosis of locally advanced PDAC after comprehensive evaluation of images and the administration of gemcitabine combined with fluorouracil as the chemotherapy regimen. The exclusion criteria were as follows: 1) having an unclear pathological diagnosis; 2) receiving neoadjuvant chemotherapy or radiotherapy prior to CEUS; 3) being critically ill and quitting treatment; 4) being pregnant or nursing; and 5) refusing to cooperate with telephone follow-up (Figure 1). In addition, clinical data, such as serum level of carbohydrate antigen 19-9 (CA19-9), were collected, and the patients' survival times were followed up.

\section{Ultrasound Technique}

A Philips iU22 (Philips Healthcare, Eindhoven, Netherlands) colour Doppler ultrasound system was used in this study. The location, size, morphology, and echo of the lesions were observed and recorded. The blood vessels of the lesions were displayed by colour Doppler ultrasound, the velocity range was set at $+10 \sim-10 \mathrm{~cm} / \mathrm{s}$, the wall filter was set at $40-50 \mathrm{~Hz}$, blood flow distribution and spectral characteristics were observed and recorded separately, and the static images were stored in JPG format.

CEUS used pulse-inversion harmonic (PIH) imaging technology, and the mechanical index (MI) was set at 0.08 .
The optimal section of the lesion was selected, and the normal pancreatic tissues were included. The focal position (single point) was set at the deepest part of the section. The ultrasound contrast agent was SonoVue (Bracco, Italy). After the establishment of peripheral venous access, a rapid bolus injection of $2.4 \mathrm{~mL}$ of contrast agent was performed, followed by rinsing with $5 \mathrm{~mL}$ of saline, and the patient was maintained as still as possible to continuously observe the dynamic perfusion process of the lesion in real-time, with an observation time of no less than $2 \mathrm{~min}$ and $30 \mathrm{~s}$. The enhanced dynamic images were saved in AVI format.

\section{Assessment of Ultrasound Enhancement Patterns}

The two sonographers (with 10 years and 5 years of CEUS experience in pancreatic imaging, respectively) independently evaluated the CEUS enhancement patterns of all enrolled patients without any knowledge of the pathology and prognosis of the enrolled subjects. Controversial cases were discussed, and consensus was reached as the final result. The enhanced images were categorized into the arterial phase (from the injection of contrast agent to $30 \mathrm{~s}$ after injection) and the venous phase (30-120 s after the injection of contrast agent), and enhancement of lesion intensity was classified as hypo-, iso-, hyper- or non-enhancement compared with normal pancreatic parenchyma from the same period. ${ }^{4}$

\section{Quantitative Analysis of CEUS by Software}

The dynamic CEUS images of each lesion were analysed using QontraXt Version 3.06 (ESAOTE Ltd.), a multiparameter quantitative analytical tool for CEUS. CEUS imaging was performed $2 \mathrm{~min}$ after the injection of contrast agent. The images contained the complete arterial and venous phases of tumour perfusion. First, the region of interest (ROI) was selected for tumour tissues and normal tissues. ROIs in tumour tissues with a rapid increase in the arterial phase and a rapid decrease in the venous phase were selected, and vessels, necrotic regions, and dilatation catheters were avoided. QontraXt software was used to examine each frame, delete frames with lesion detachment from the ROI due to patient respiratory movement, and obtain the preliminary time-intensity curves (TICs) of normal pancreatic tissues and tumour tissues. Gamma curve fitting of the bolus injection was performed on the preliminary TICs to obtain the final TICs of normal pancreatic tissues and tumour tissue, and various TIC parameters of the tumour tissues and 
Patients received ultrasound-guided biopsy of pancreatic space-occupying

lesions between January 2014 and December 2017( $n=56)$

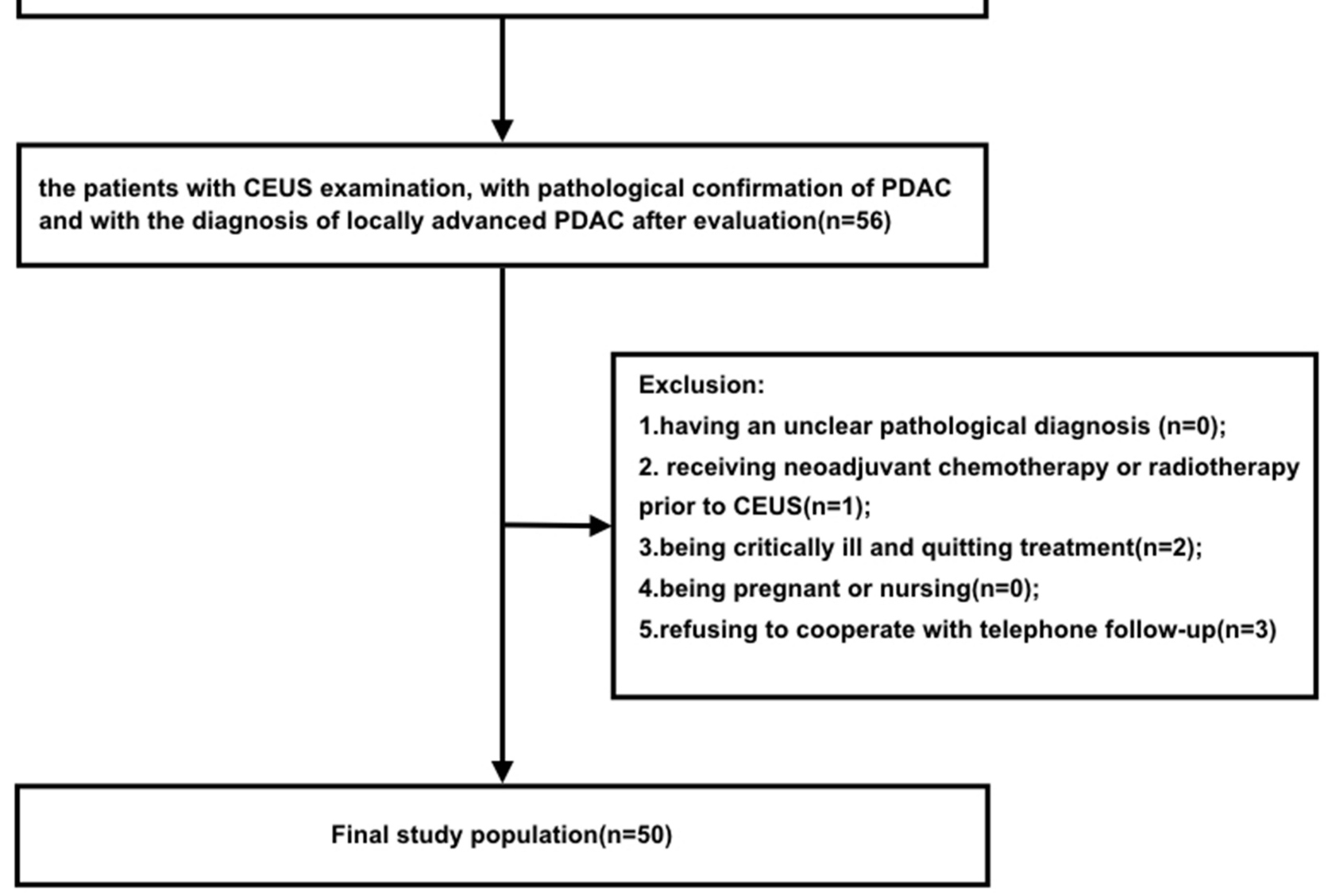

Figure I Flow diagram of patient inclusion and exclusion.

normal tissues during perfusion were obtained, including the peak intensity during tumour perfusion (peak $\mathrm{t}_{\text {tumour }}$ ) and normal tissue perfusion ( peak $_{\text {normal }}$ ); the curve sharpness during tumour perfusion (sharpness tumour $_{\text {) }}$ and normal tissue perfusion ( sharpness $\left._{\text {normal }}\right)$; the time to peak during tumour perfusion $\left(\mathrm{TP}_{\text {tumour }}\right)$ and normal tissue perfusion $\left(\mathrm{TP}_{\text {normal }}\right)$; and the area under the curve during tumour perfusion $\left(\mathrm{AUC}_{\text {tumour }}\right)$ and normal tissue perfusion $\left(\mathrm{AUC}_{\text {normal }}\right)$.

The two sets of parameters were compared to obtain other relevant parameters as follows: ${ }^{5}$

Absolute enhanced peak intensity (AEpeak) = peak $_{\text {normal }}$ - peak tumour $_{\text {; }}$

Relative enhanced peak intensity (REpeak) = ( peak $_{\text {normal }}$ - peak $\left.k_{\text {tumour }}\right) /$ peak $_{\text {normal }}$;

Absolute time to peak (AETP) $=\mathrm{TP}_{\text {normal }}-\mathrm{TP}_{\text {tumour }}$;

Relative time to peak $(\mathrm{RETP})=\left(\mathrm{TP}_{\text {normal }}-\mathrm{TP}_{\text {tumour }}\right) /$ $\mathrm{TP}_{\text {normal }}$;

Absolute curve sharpness (AEsharpness) = sharpness normal $_{\text {- sharpness }}$ tumour;
Relative curve sharpness (REsharpness) = ( sharpness $_{\text {normal }}$ - sharpness $_{\text {tumour }}$ )/ sharpness $_{\text {normal }}$;

Absolute area under the curve $(\mathrm{AEAUC})=\mathrm{AUC}_{\text {normal }}$ $\mathrm{AUC}_{\text {tumour}}$;

Relative area under the curve $($ REAUC $)=\left(\mathrm{AUC}_{\text {normal }}\right.$ $\left.\mathrm{AUC}_{\text {tumour }}\right) / \mathrm{AUC}_{\text {normal }}$.

The above curves and related parameters were saved for analysis and comparison.

\section{Statistical Analysis}

The data in this study were analysed using the statistical software SPSS 21.0. Patient survival was determined from the date of first imaging evaluation to the most recent follow-up examination or patient death. For data conforming to a normal distribution, comparisons between two independent groups were performed using the $t$-test; if the data did not conform to a normal distribution, the median values (quartiles) were used, and comparisons between two groups were performed using the Mann- 
Whitney $U$-test. Intergroup comparisons were performed using chi-square analysis, correlations between two variables were analysed using Spearman rank correlation analysis, and log-rank analysis was used for intergroup comparisons of survival. $\mathrm{P}<0.05$ was regarded as indicative of statistical significance.

\section{Results}

\section{Population: Clinical Outcome $(n=56)$}

A total of 56 pancreatic cancer patients (35 males and 21 females) were included in this study; these patients' clinical data are shown in Table 1.

\section{Ultrasound Findings in the Overall Population $(n=56)$}

The mean maximum diameter of tumour tissues measured by greyscale ultrasound was $4.74 \pm 1.23 \mathrm{~cm}$, with a median of $4.50 \mathrm{~cm} ; 33.9 \%(19 / 56)$ of the tumours were located in the head of the pancreas; $35.7 \%(20 / 56)$ were located in the pancreas, and $30.4 \%(17 / 56)$ were located in the pancreatic tail. All (56/56) lesions showed poorly defined focal hypoechoic regions, and 14.3\% (8/56) of the lesions had necrotic regions.

Colour Doppler ultrasound showed that clear blood flow signals were not detected in $76.8 \%(43 / 56)$ of the lesions, and $23.2 \%$ (13/56) showed some blood flow signals.

CEUS dynamic image analysis showed that 100\% (56/ 56) of the PDAC lesions exhibited a rapid increase in the arterial phase and a rapid decline in the venous phase; however, the enhancement pattern in the arterial phase was divided into diffuse iso-enhancement and hypoenhancement. The former, which accounted for $30.3 \%$ of all lesions (17/56), showed relatively uniform diffuse enhancement in the arterial phase and enhancement intensity similar to that of normal pancreatic parenchyma; the latter, which accounted for $69.6 \%$ of all lesions (39/56), showed relatively uniform low enhancement or peripheral ring enhancement in the arterial phase and overall enhancement intensity significantly lower than that of the normal pancreas (Figure 2).

There were no significant differences between the two groups (two enhancement patterns) in patient sex, age at disease onset, maximum tumour diameter, CA19-9 level or colour Doppler blood flow signal characteristics $(\mathrm{P}>0.05)$ (Table 1).

\section{Comparative Analysis of CEUS Enhancement Pattern and Tumour Tissue Differentiation $(n=26)$}

In this study, the pathological results of 56 PDAC cases were obtained through ultrasound-guided percutaneous pancreatic biopsy. Among them, 46 patients underwent fine-needle aspiration (FNA) with a 20G needle, and 10 patients underwent coarse needle biopsy (four cases also underwent FNA). It is worth noting that in this study, multi-point puncture was tried to avoid deviation caused by heterogeneity.

Biopsy indicated clear differentiation of the tumour tissue in 26 cases, of which $46.2 \%(12 / 26)$ were well differentiated and $53.8 \%(14 / 26)$ were moderately to poorly differentiated. Among the 26 cases, 10 cases had diffuse iso-enhancement (38.5\%), and 16 cases had hypoenhancement in the arterial phase $(61.5 \%)$. There were no statistically significant differences $(\mathrm{P}>0.05)$ with respect to sex, age at disease onset, lesion size, CA19-9 level, and blood flow signals and enhancement pattern between the well-differentiated and moderately to poorly differentiated groups (Table 1).

\section{Comparative Analysis of CEUS Enhancement Pattern and Survival ( $\mathrm{n}=$ 50)}

Some patients were excluded from the comparative analysis of CEUS enhancement patterns because they were lost to follow-up survival time $(\mathrm{n}=6)$. Fifty patients were eventually included, of whom $72 \%$ (36/50) showed hypoenhancement, with the remaining 28\% (14/50) showing iso-enhancement. There were no statistically significant differences in sex, age at disease onset, maximum tumour diameter, CA19-9 level and tumour blood flow signals between the groups with different enhancement patterns $(\mathrm{P}>0.05)$ (Table 2).

The follow-up results (ending date of February 2019) showed that 39 patients died due to the tumour (78\%) and 11 patients survived $(22 \%)$. The median survival time in the hypo-enhancement group was $321.0 \pm 205.5$ days, and the median survival time in the iso-enhancement group was $627.1 \pm 311.6$ days (Table 3 ). The difference in survival time between the two groups was statistically significant ( $\mathrm{P}=0.004<0.05$, as shown in Figure 3), and the survival time in the iso-enhancement group was significantly longer than that in the hypo-enhancement group. 


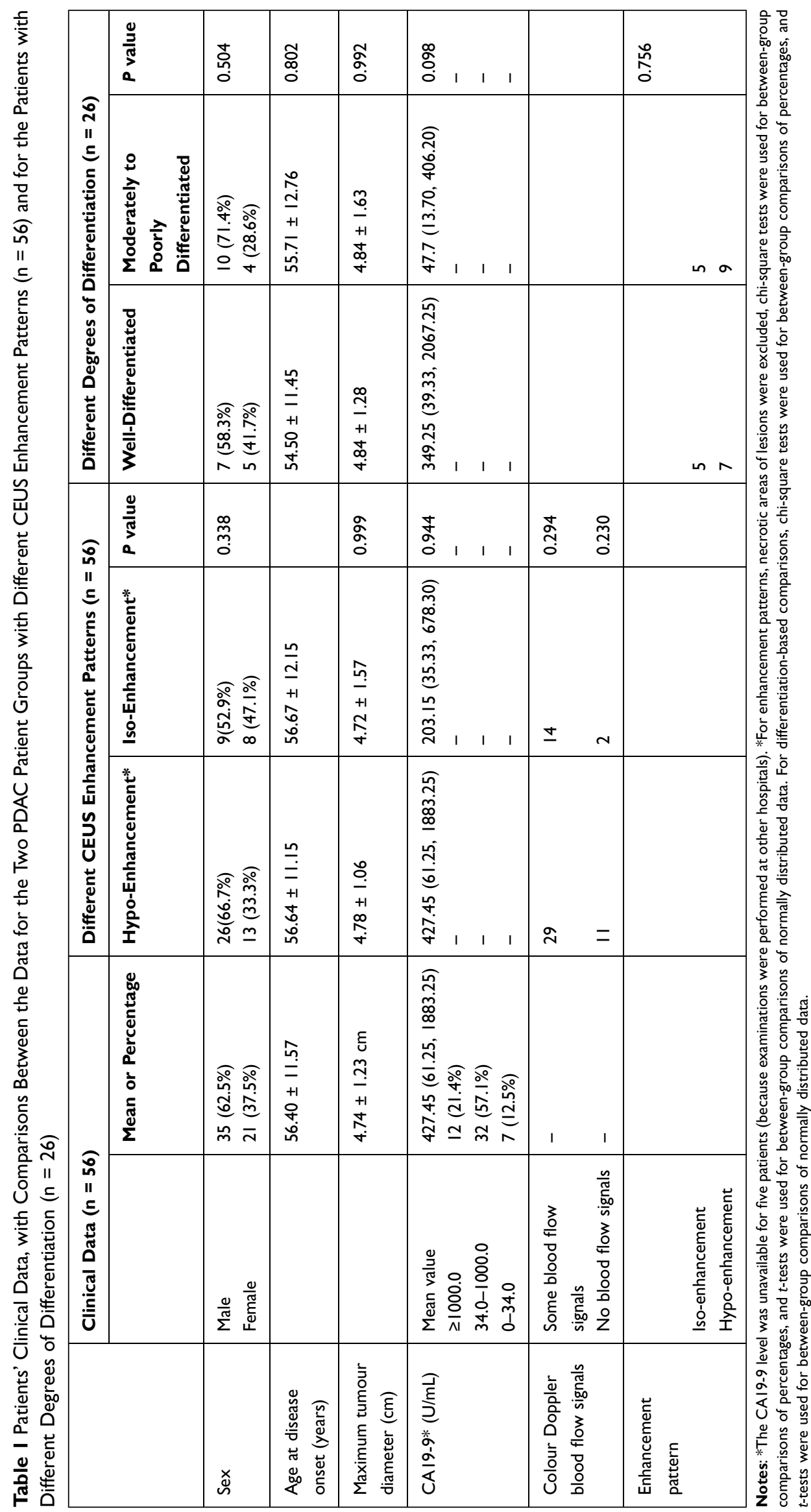


A

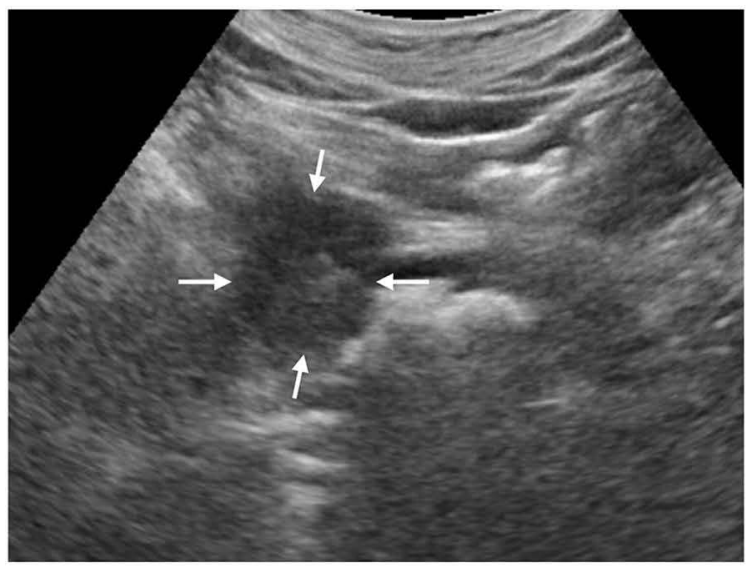

C

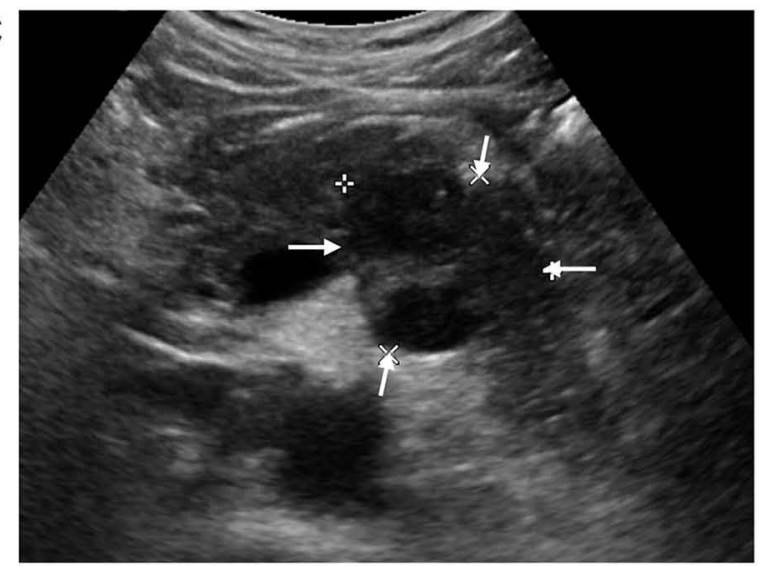

B

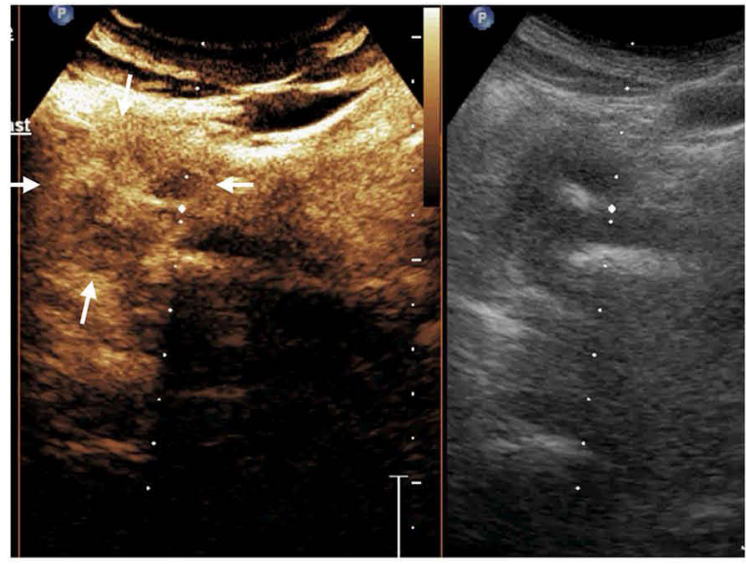

D

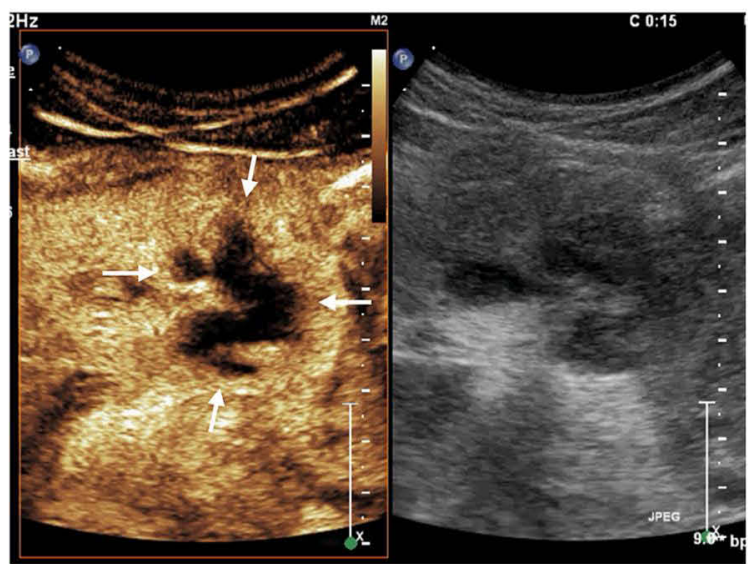

Figure 2 CEUS enhancement patterns in the arterial phase in PDAC. (A and B): A 53-year-old male patient with PDAC located in the head of the pancreas. Rapid iso-enhancement was observed in the arterial phase of CEUS ( $18 \mathrm{~s}$ ) (as shown by the white arrows). (C and D): A 42 year-old male patient with PDAC located in the body of the pancreas. Hypoenhancement was observed in the arterial phase of CEUS (I5 s), and no areas of enhancement were visible within the tumour (as shown by the white arrows).

\section{Analysis of TIC Parameters of CEUS} Comparison Analysis of TIC Parameters and CEUS Enhancement Patterns of PDAC

The TIC parameters of different CEUS enhancement patterns of PDAC were compared, and the results are shown in Table 4. The results showed that peak tumour, sharpness $_{\text {tumour, }}$ AEpeak, AEsharpness, and REsharpness differed significantly between the hypo- and isoenhancement groups $(\mathrm{P}<0.05)$, while the remaining parameters did not $(P>0.05)$. In addition, the levels of peak $_{\text {tumour, }}$ sharpness tumour, $_{\text {AEsharpness, and }}$ REsharpness of the iso-enhancement group were significantly higher than those in the hypo-enhancement group.

Table 2 Comparison of Sex, Age at Disease Onset, Size of Lesions, and CA19-9 Level for PDAC Patients with Different Enhancement Patterns

\begin{tabular}{|l|l|l|l|l|}
\hline & Hypo-Enhancement* & Iso-Enhancement* & X2/T & P value \\
\hline $\begin{array}{l}\text { Sex } \\
\text { Female } \\
\text { Male }\end{array}$ & $\begin{array}{l}13(36.1 \%) \\
23(63.9 \%)\end{array}$ & $\begin{array}{l}7(50 \%) \\
7(50 \%)\end{array}$ & 0.81 & 0.368 \\
\hline Age at disease onset (years) & $56.39 \pm 11.47$ & $56.43 \pm 12.26$ & -0.011 & -0.222 \\
\hline Maximum tumour diameter (cm) & $4.63 \pm 0.97$ & $4.71 \pm 1.25$ & 0.991 \\
\hline CAl9-9 (U/mL) & $427.45(61.25,1883.25)$ & $203.15(35.33,678.30)$ & 0.982 & 0.825 \\
\hline
\end{tabular}

Notes: *For enhancement patterns, necrotic areas of lesions were excluded, chi-square tests were used for between-group comparisons of percentages, and $t$-tests were used for between-group comparisons of normally distributed data. 
Table 3 Survival of PDAC Patients with Different Enhancement Patterns Based on Follow-Up Results

\begin{tabular}{|l|l|l|l|l|l|}
\hline Enhancement Pattern & Number of Survivors & Number of Deaths & Median Survival Time (Days) & Chi-Square & $P$ value \\
\hline Hypo-enhancement & 7 & 29 & $321.0 \pm 205.5$ & 8.142 & 0.004 \\
Iso-enhancement & 4 & 10 & $627.1 \pm 311.6$ & \\
\hline
\end{tabular}

Note: Cox regression was used.

\section{Discussion}

CEUS has certain advantages for the qualitative diagnosis of pancreatic space-occupying lesions ${ }^{3,6-8}$ and exhibits high sensitivity and specificity in distinguishing between pancreatic cancer and pancreatic neuroendocrine tumours. ${ }^{9,10}$ When CT cannot allow for clear diagnosis of a lesion, CEUS can provide additional imaging information to facilitate diagnosis. CEUS can help determine the targeted area of biopsy to improve biopsy accuracy. ${ }^{4,11}$ The enhancement pattern and intensity of lesions can be evaluated by CEUS quantitative analysis software. ${ }^{12-14}$ Therefore, CEUS is a reliable method to evaluate pancreatic lesions.

Previous studies have reported that compared with normal pancreatic parenchyma, the CEUS enhancement in most pancreatic cancer lesions is hypo-enhancement. ${ }^{4}$ Using this particular CEUS enhancement pattern, the diagnostic accuracy can reach as high as $87.8 \%,{ }^{15}$ and this specific CEUS enhancement of PDAC is usually used for differential diagnosis in clinical practice. In recent years, some studies have found that different enhancement

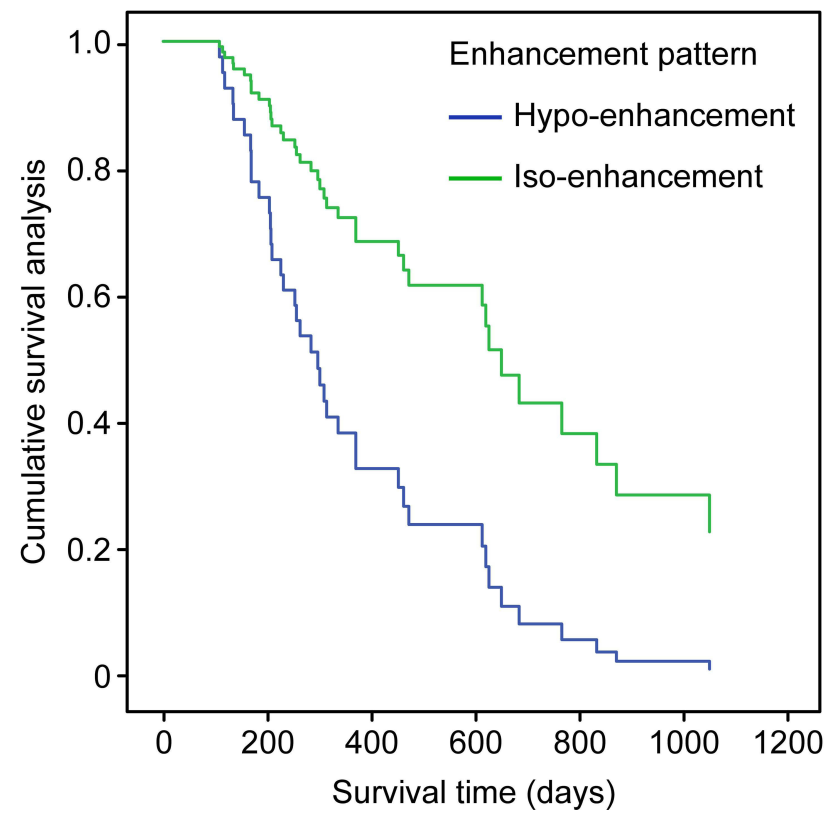

Figure 3 Survival analysis of the hypo- and iso-enhancement groups. patterns of PDAC are associated with patient prognosis. $^{5,16,17}$ Lee et al ${ }^{17}$ conducted a study on MRI for pancreatic cancer and found that patients with uniform diffuse enhancement in their lesions had longer survival times than patients with only peripheral enhancement and no central enhancement in their lesions. Currently, such studies are limited to CT and MRI. Similar studies on CEUS are rare, and only one CEUS study, by Akasu et al ${ }^{18}$ in 2012, has been reported. The results showed that the survival time of the hypo-enhancement group was significantly shorter than that of the hyper-enhancement group, but only 16 cases were included in the study. ${ }^{18}$

This study showed that the CEUS enhancement of PDAC lesions in the arterial phase exhibited two patterns, iso-enhancement and hypo-enhancement. The differences in sex, age at disease onset, lesion size, and lesion blood flow between the different enhancement patterns were not significant, but the enhancement pattern was significantly correlated with patient survival time. The survival time was significantly longer in the iso-enhancement group than in the hypo-enhancement group. In this study, image processing software was used to analyse the entire process of CEUS enhancement to confirm the objective differences between the two enhancement patterns. The differences in TIC parameters (peak $_{\text {tumour, sharpness }}$ tumour, AEpeak, AEsharpness, and REsharpness) between the different enhancement patterns were significant. This difference can be simply described as "high and sharp" for the TIC curves of the iso-enhancement group compared with the hypo-enhancement group.

Pancreatic cancer is often accompanied by an inflammatory reaction in peripheral tissues, and previous studies have shown that inflammatory reaction and PDAC can be differentiated by different CEUS enhancement features, ie, the iso-enhancement pattern occurs over the whole process of CEUS for inflammatory lesions, while the enhancement in pancreatic cancer declines rapidly in the venous phase. In this study, the selected ROIs in the tumour tissues were the regions with a rapid increase in the arterial phase and a rapid decline in the venous phase, and regions with similar rates of increase and decrease as normal tissues 
Table 4 Comparison of TIC Parameters Between Different CEUS Enhancement Patterns of PDAC

\begin{tabular}{|c|c|c|c|}
\hline & Hypo-Enhancement & Iso-Enhancement & $P$ value \\
\hline Peak normal $_{1}$ & $59.77 \pm 12.3$ & $55.03 \pm 11.88$ & 0.849 \\
\hline $\mathrm{TP}_{\text {normal }}(\mathrm{ms})$ & $32,198.5(22,288,35,984.5)$ & $27,215(24,039,34,010)$ & 0.320 \\
\hline Sharpness $_{\text {normal }}(1 / s)$ & $0.07(0.04,0.11)$ & $0.06(0.04,0.08)$ & 0.604 \\
\hline $\mathrm{AUC}_{\text {normal }}(\mathrm{l} / \mathrm{s})$ & $4.15(2.55,5.95)$ & $2.95(2.1,4.1)$ & 0.170 \\
\hline Peak $_{\text {tumour }}$ & $38.9(35.8,44.1)$ & $49.4(46,59.4)$ & $<0.001$ \\
\hline $\mathrm{TP}_{\text {tumour }}(\mathrm{ms})$ & $26,706.33 \pm \mid 2,701.85$ & $28,119.14 \pm 7769.61$ & 0.235 \\
\hline Sharpness $_{\text {tumour }}(1 / \mathrm{s})$ & $0.12(0.09,0.22)$ & $0.08(0.06,0.12)$ & 0.006 \\
\hline$A \cup C_{\text {tumour }}(\mathrm{I} / \mathrm{s})$ & $4.9(2.95,8)$ & $4.1(3.1,5.6)$ & 0.239 \\
\hline AEpeak & $20.45 \pm 11.11$ & $2.51 \pm 6.06$ & 0.013 \\
\hline REpeak & $0.33 \pm 0.16$ & $0.03 \pm 0.10$ & 0.288 \\
\hline AETP (ms) & $3685(-3902,9994)$ & $613(-5147,4503)$ & 0.202 \\
\hline RETP & $0.13(-0.19,0.37)$ & $0.02(-0.22,0.13)$ & 0.195 \\
\hline AEsharpness (I/s) & $-0.07(-0.14,-0.04)$ & $-0.02(-0.04,0)$ & 0.001 \\
\hline REsharpness & $-1.06(-2.37,-0.5 \mathrm{I})$ & $-0.29(-0.91,0.05)$ & 0.009 \\
\hline AEAUC (I/s) & $-1.12 \pm 3.34$ & $-0.53 \pm 2.19$ & 0.273 \\
\hline REAUC & $-0.2(-1.33,0.09)$ & $-0.42(-0.85,0.04)$ & 0.897 \\
\hline
\end{tabular}

Notes: For between-group comparisons, $t$-tests and the Mann-Whitney $U$-test were used for normally and non-normally distributed data, respectively.

were carefully avoided to prevent the inclusion of ROIs with inflammatory reaction regions and ensure the reliability of the results. The enhanced tumour blood vessels inside the PDAC lesions revealed by CEUS are an important component of the tumour microenvironment, ${ }^{19}$ and different enhancement patterns of pancreatic cancer may be directly associated with pancreatic cancer angiogenesis and fibrosis. $^{20}$

Many studies have confirmed that CEUS has significant advantages in evaluating and quantifying the angiogenesis and distribution characteristics of PDAC. ${ }^{21,22}$ In the study by Akasu et al, ${ }^{18}$ the CEUS enhancement intensity was positively correlated with microvessel density (MVD), and CEUS could be used to evaluate the MVD of pancreatic cancer. D'Onofrio et al ${ }^{23}$ suggested that CEUS can accurately describe the angiogenesis of pancreatic cancer, and CEUS enhancement was also positively correlated with histological grade and MVD. In addition, Erkan et $\mathrm{al}^{24}$ found that the presence of a large amount of matrix surrounding the pancreatic cancer cells results in low MVD in tumour tissue, and the wrapping of the matrix on the tumour cells generates a hypoxic microenvironment in pancreatic cancer cells, thereby promoting proliferation, inhibiting apoptosis, and enhancing the invasive capability of cancer cells. $^{24-26}$ Kuwahara et $\mathrm{al}^{27}$ found that the survival time of tumour patients with high expression of fibroblast growth factor-2 (FGF-2) was significantly shorter than that of patients with low expression or no expression. Zhu et $\mathrm{al}^{5}$ showed that pancreatic cancer patients with poor enhancement often had a high degree of fibrosis and a poor prognosis.

The above evidence suggests that the enhancement information of PDAC lesions provided by CEUS can be used as a reliable basis for evaluating tumour angiogenesis and the degree of fibrosis. In this study, the isoenhancement pattern indicated that tumour angiogenesis was abundant and uniformly distributed in the lesion, with a relatively low degree of fibrosis. The hypoenhancement pattern was associated with sparse and unevenly distributed tumour angiogenesis and a relatively high degree of fibrosis. The application of different enhancement patterns in the prognostic evaluation of PDAC can provide a basis for risk stratification before clinical treatment. Further studies are needed to determine whether the sensitivity of PDAC lesions with different enhancement patterns to chemotherapy differs or whether different treatment regimens can be selected.

This study also has some limitations. Most patients failed to complete all treatment and follow-up in our hospital, and the survival information was difficult to collect. Therefore, the number of PDAC patients included in this study was small, and further in-depth studies are needed. Although most of the patients in this study received standardized chemotherapy with gemcitabine combined with fluorouracil, with the progression of the 
disease, it was difficult to accurately record and classify patients after the combined use of other treatments, which may have had some impact on the patient survival analysis. This study did not include an evaluation of the sensitivity and efficacy of chemotherapy by CEUS, and relevant studies should be conducted in the future.

\section{Conclusion}

CEUS enhancement in PDAC could be classified into isoenhancement and hypo-enhancement patterns in the arterial phase, and patients in the iso-enhancement group had a longer survival time. CEUS is recommended for observing the enhancement pattern of PDAC and may allow for risk stratification before treatment and evaluations of efficacy based on TIC parameters.

\section{Abbreviations}

CEUS, contrast-enhanced ultrasound; PDAC, pancreatic ductal adenocarcinoma; OS, overall survival; CA19-9, carbohydrate antigen 19-9; TIC, time-intensity curve; peak, peak intensity; sharpness, curve sharpness; TP, time to peak; AUC, area under the curve; ROI, region of interest.

\section{Ethics Approval and Informed Consent}

Due to the retrospective nature of the study, ethics approval was waived by our ethics board.

\section{Funding}

The work was supported by the Chinese Academy of Medical Sciences Innovation Fund for Medical Sciences (2016-I2M-3-005 \& 2020-I2M-C\&T-B-039) and the National Natural Science Foundation of China (grant No. 81873902$)$.

\section{Disclosure}

The authors of this manuscript declare no relationships with any companies, whose products or services may be related to the subject matter of the article. The authors report no conflicts of interest in this work.

\section{References}

1. Miller KD, Siegel RL, Lin CC, et al. Cancer treatment and survivorship statistics, 2016. CA Cancer J Clin. 2016;66(4):271-289. doi:10.3322/caac. 21349

2. Conlon KC, Klimstra DS, Brennan MF. Long-term survival after curative resection for pancreatic ductal adenocarcinoma. Clinicopathologic analysis of 5-year survivors. Ann Surg. 1996;223 (3):273-279. doi:10.1097/00000658-199603000-00007
3. Lin LZ, Li F, Liu Y, Xing LX, Du LF. Contrast-enhanced ultrasound for differential diagnosis of pancreatic mass lesions: a meta-analysis. Med Ultrason. 2016;18(2):163-169. doi:10.11152/mu.2013.2066.182.ceu

4. Sidhu PS, Cantisani V, Dietrich CF, et al. The EFSUMB guidelines and recommendations for the clinical practice of contrast-enhanced ultrasound (CEUS) in non-hepatic applications: update 2017 (short version). Ultraschall Med. 2018;39(02):154-180. doi:10.1055/s-0044-101254

5. Zhu L, Shi X, Xue H, et al. CT imaging biomarkers predict clinical outcomes after pancreatic cancer surgery. Medicine (Baltimore). 2016;95(5):e2664. doi:10.1097/MD.0000000000002664

6. Gong TT, Hu DM, Zhu Q. Contrast-enhanced EUS for differential diagnosis of pancreatic mass lesions: a meta-analysis. Gastrointest Endosc. 2012;76(2):301-309. doi:10.1016/j.gie.2012.02.051

7. D'Onofrio M, Biagioli E, Gerardi C, et al. Diagnostic performance of contrast-enhanced ultrasound (CEUS) and contrast-enhanced endoscopic ultrasound (ECEUS) for the differentiation of pancreatic lesions: a systematic review and meta-analysis. Ultraschall Med. 2014;35(06):515-521. doi:10.1055/s-0034-1385068

8. Iglesias-Garcia J, Lindkvist B, Larino-Noia J, Abdulkader-Nallib I, Dominguez-Munoz JE. Differential diagnosis of solid pancreatic masses: contrast-enhanced harmonic (CEH-EUS), quantitative-elastography (QE-EUS), or both? Uni Eur Gastroenterol J. 2017;5(2):236-246. doi:10.1177/2050640616640635

9. Leem G, Chung MJ, Park JY, et al. Clinical value of contrast-enhanced harmonic endoscopic ultrasonography in the differential diagnosis of pancreatic and gallbladder masses. Clin Endosc. 2018;51(1):80-88. doi:10.5946/ce.2017.044

10. Rickes S, Monkemuller K, Malfertheiner P. Contrast-enhanced ultrasound in the diagnosis of pancreatic tumors. JOP. 2006;7:584-592.

11. Beyer-Enke SA, Hocke M, Ignee A, Braden B, Dietrich CF. Contrast enhanced transabdominal ultrasound in the characterisation of pancreatic lesions with cystic appearance. JOP. 2010;11:427-433.

12. D’Onofrio M, Canestrini S, Crosara S, De Robertis R, Pozzi Mucelli R. Contrast enhanced ultrasound with quantitative perfusion analysis for objective characterization of pancreatic ductal adenocarcinoma: a Feasibility Study. World J Radiol. 2014;6(3):31-35. doi:10.4329/wjr.v6.i3.31

13. Kitano M, Kamata $\mathrm{K}$, Imai $\mathrm{H}$, et al. Contrast-enhanced harmonic endoscopic ultrasonography for pancreatobiliary diseases. Dig Endosc. 2015;27(Suppl 1):60-67. doi:10.1111/den.12454

14. Kersting S, Konopke R, Kersting F, et al. Quantitative perfusion analysis of transabdominal contrast-enhanced ultrasonography of pancreatic masses and carcinomas. Gastroenterology. 2009;137 (6):1903-1911. doi:10.1053/j.gastro.2009.08.049

15. D'Onofrio M, Barbi E, Dietrich CF, et al. Pancreatic multicenter ultrasound study (PAMUS). Eur J Radiol. 2012;81(4):630-638. doi:10.1016/j.ejrad.2011.01.053

16. Yoon SH, Lee JM, Cho JY, et al. Small $(</=20 \mathrm{~mm})$ pancreatic adenocarcinomas: analysis of enhancement patterns and secondary signs with multiphasic multidetector CT. Radiology. 2011;259:442-452. doi:10.1148/radiol.11101133

17. Lee S, Kim SH, Park HK, Jang KT, Hwang JA, Kim S. Pancreatic ductal adenocarcinoma: rim enhancement at MR imaging predicts prognosis after curative resection. Radiology. 2018;288(2):456-466. doi:10.1148/radiol.2018172331

18. Akasu G, Kawahara R, Yasumoto M, et al. Clinicopathological analysis of contrast-enhanced ultrasonography using perflubutane in pancreatic adenocarcinoma. Kurume Med J. 2012;59(3.4):45-52. doi: $10.2739 /$ kurumemedj.59.45

19. Catalano V, Turdo A, Di Franco S, Dieli F, Todaro M, Stassi G. Tumor and its microenvironment: a synergistic interplay. Semin Cancer Biol. 2013;23(6):522-532. doi:10.1016/j.semcancer.2013.08.007

20. Hata H, Mori H, Matsumoto S, et al. Fibrous stroma and vascularity of pancreatic carcinoma: correlation with enhancement patterns on CT. Abdom Imaging. 2010;35(2):172-180. doi:10.1007/s00261-0089460-0 
21. D’Onofrio M, Malago R, Zamboni G, et al. Contrast-enhanced ultrasonography better identifies pancreatic tumor vascularization than helical CT. Pancreatology. 2005;5(4-5):398-402. doi:10.1159/000086540

22. Nishida M, Koito K, Hirokawa N, Hori M, Satoh T, Hareyama M. Does contrast-enhanced ultrasound reveal tumor angiogenesis in pancreatic ductal carcinoma? A prospective study. Ultrasound Med Biol. 2009;35(2):175-185. doi:10.1016/j.ultrasmedbio.2008.08.008

23. D’Onofrio M, Zamboni GA, Malago R, et al. Resectable pancreatic adenocarcinoma: is the enhancement pattern at contrast-enhanced ultrasonography a pre-operative prognostic factor? Ultrasound Med Biol. 2009;35(12):1929-1937. doi:10.1016/j.ultrasmedbio.2009.06.1100

24. Erkan M, Kleeff J, Gorbachevski A, et al. Periostin creates a tumor-supportive microenvironment in the pancreas by sustaining fibrogenic stellate cell activity. Gastroenterology. 2007;132 (4):1447-1464. doi:10.1053/j.gastro.2007.01.031
25. Erkan M. The role of pancreatic stellate cells in pancreatic cancer. Pancreatology. 2013;13(2):106-109. doi:10.1016/j.pan.2013.01.008

26. Fujita H, Ohuchida K, Mizumoto K, et al. alpha-smooth muscle actin expressing stroma promotes an aggressive tumor biology in pancreatic ductal adenocarcinoma. Pancreas. 2010;39(8):1254-1262. doi:10.1097/MPA.0b013e3181dbf647

27. Kuwahara K, Sasaki T, Kuwada Y, Murakami M, Yamasaki S, Chayama K. Expressions of angiogenic factors in pancreatic ductal carcinoma: a correlative study with clinicopathologic parameters and patient survival. Pancreas. 2003;26(4):344-349. doi:10.1097/ 00006676-200305000-00006

\section{Publish your work in this journal}

Cancer Management and Research is an international, peer-reviewed open access journal focusing on cancer research and the optimal use of preventative and integrated treatment interventions to achieve improved outcomes, enhanced survival and quality of life for the cancer patient.
The manuscript management system is completely online and includes a very quick and fair peer-review system, which is all easy to use. Visit http://www.dovepress.com/testimonials.php to read real quotes from published authors. 